\title{
Impacto de las Condiciones Sociodemográficas y Orales en la Calidad de Vida Relacionada a la Salud Oral en Preescolares de Temuco, Chile
}

\author{
Impact of Sociodemographic and Oral Conditions on Oral Health \\ Related Quality of Life in Preschool Children From Temuco, Chile
}

\author{
Núñez-Contreras, Javiera ${ }^{1,2}$; Hofer-Durán, Paulina²; Sinsay-Schmeisser, Javiera ${ }^{3}$ \& Zaror, Carlos ${ }^{4,5}$
}

\begin{abstract}
NÚÑEZ-CONTRERAS, J.; HOFER-DURÁN, P.; SINSAY-SCHMEISSER, J. \& ZAROR, C. Impacto de las condiciones sociodemográficas y orales en la calidad de vida relacionada a la salud oral en preescolares de Temuco, Chile. Int. J. Odontostomat., 15(2):503-512, 2021.

RESUMEN: Las condiciones orales son altamente prevalentes en preescolares y afectan la calidad de vida relacionada con la salud oral (CVRSO) de quienes la padecen. El objetivo de este estudio fue determinar el impacto de las variables sociodemográficas y clínicas en la CVRSO en preescolares de la Comuna de Temuco, Chile. Se realizó un estudio de corte transversal en preescolares entre 3 a 5 años inscritos en los jardines de Fundación INTEGRA de la ciudad de Temuco, Chile. Cuatro investigadores calibrados evaluaron la prevalencia de caries, maloclusiones, traumatismo dentoalveolar, hipoplasias anteriores y posteriores, presencia de placa y gingivitis. La CVRSO fue medida de la versión en español de la Early Childhood Oral Health Impact Scale (ECOHIS). Adicionalmente los padres completaron un cuestionario sobre los antecedentes sociodemográficos del niño. La asociación bivariada entre las variables clínicas y sociodemográficas con CVRSO fue realizada a través de las pruebas no paramétricas de Kruskal-Wallis o Mann Whitney. Posteriormente se realizó un análisis multivariado utilizando un modelo lineal generalizado para asociar los diferentes factores con CVRSO. Fueron incluidos 202 preescolares (105 niños y 97 niñas) con edad promedio de 3,3 (SD 0,6). La puntuación total de ECOHIS tuvo una media de 2,60 (SD 4,64). El análisis multivariado mostró que el estado civil viudo(a) ( $P R=2,93 ; p=<0,001)$ y un ceod $>$ a $5(P R=7,16 ; p=<0,001)$, generan un impacto negativo sobre CVRSO. No se encontró asociación con otras variables sociodemográficas y clínicas. Este estudio concluye que existe una asociación entre la presencia de un ceod > 5 y el vivir con cuidadores viudos en la disminución de la CVRSO de los preescolares.
\end{abstract}

PALABRAS CLAVE: Salud oral, calidad de vida, preescolares.

\section{INTRODUCCIÓN}

Las enfermedades que afectan la cavidad oral son altamente prevalentes a pesar de la indiscutible mejora de las últimas décadas (Kassebaum et al., 2017). La caries dental es considerada la enfermedad crónica más prevalente a nivel oral, afectando a 573 millones de niños a nivel mundial (Kassebaum et al.). En Chile, las patologías orales siguen siendo un importante problema de salud pública, reportándose prevalencias que pueden superar el $60 \%$ en prescolares (Ministerio de Salud, 2017; Espinoza-

\footnotetext{
${ }^{1}$ Estudiante Magíster en Odontología, Universidad de La Frontera, Temuco, Chile.

${ }^{2}$ Escuela de Odontología, Universidad de La Frontera, Temuco, Chile.

${ }^{3}$ Cirujano Dentista, Universidad de La Frontera. Temuco, Chile.

${ }^{4}$ Departamento de Odontopediatría y Ortodoncia, Universidad de La Frontera, Temuco, Chile

${ }^{5}$ Centro de Investigación en Epidemiología, Economía y Salud Pública Oral (CIEESPO), Facultad de Odontología, Universidad de La Frontera, Temuco, Chile.

Financiado parcialmente a través del Concurso de Profesores patrocinantes de alumnos de pre y posgrado, otorgado por la Universidad de La Frontera y por el Programa de Magíster en Odontología de la Universidad de La Frontera, Chile.
}

Received: 2020-09-07 Accepted: 2020-12-22 
NÚÑEZ-CONTRERAS, J.; HOFER-DURÁN, P.; SINSAY-SCHMEISSER, J. \& ZAROR, C. Impacto de las condiciones sociodemográficas y orales en la calidad de vida relacionada a la salud oral en preescolares de Temuco, Chile. Int. J. Odontostomat., 15(2):503-512, 2021.

Espinoza et al.). La prevalencia de gingivitis alcanza el $67 \%$ (Ministerio de Salud). Las anomalías dentomaxilares (ADM) por otro lado, constituyen una de las alteraciones con más alta prevalencia, ubicada en el tercer lugar a nivel mundial por la OMS, afectando en Chile a un $53 \%$ de los niños de 12 años (Ministerio de Salud). Por su parte los traumatismos dentoalveolares (TDA) son considerados la $5^{\circ}$ condición más prevalente a nivel mundial (Petti et al., 2018a,b) y se estima además que sobre 1 billón de personas ha sufrido TDA en los últimos 20 años. Las alteraciones del desarrollo del esmalte (DDE) en dientes primarios, oscilan entre el 12,7\% al 49,6 \% (Jans-Muñoz et al., 2011). Está bien fundamentado que las consecuencias de los problemas de salud oral son graves, produciendo una serie de alteraciones como dolor, dificultades funcionales, desórdenes de salud general, problemas psicológicos, hospitalizaciones y atenciones de urgencia, lo que conlleva finalmente a una menor calidad de vida de los afectados (Dimberg et al., 2015; Zaror et al., 2017; Nora et al., 2018).

La Calidad de vida es reconocida como un parámetro válido en la evaluación del paciente en casi todos los ámbitos de la salud física y mental, incluida la salud oral (WHOQOL Group, 1995). En respuesta a esto, la calidad de vida relacionada con la salud oral (CVRSO), ha tomado gran relevancia, definiendo metas y resultados de tratamientos, basados en las experiencias y perspectivas de los pacientes, incorporando factores biológicos, sociales, psicológicos y culturales, reconociendo los efectos de factores contextuales como lo son los factores socioculturales, educación, estructura familiar y el acceso a la atención sobre las percepciones de salud oral (Sischo \& Broder, 2011). La evaluación de la CVRSO, ha sido incorporada como un método de vigilancia, facilitando las comparaciones de morbilidad entre problemas de salud, pudiendo establecer en el tiempo estrategias sanitarias prioritarias (Chaffee et al., 2017), sin embargo en preescolares tiene ciertos sesgos debido a las limitaciones en sus habilidades cognitivas y de comunicación de sus percepciones, lo que se puede traducir en una subestimación del impacto de los problemas de salud oral de los niños. Es por esta razón que la administración a través de un proxy es el estándar para medir CVRSO en niños en edad preescolar (Barbosa \& Gavião, 2008). Una revisión sistemática identificó cinco instrumentos para medir la CVRSO de niños en preescolares, siendo la ECOHIS el instrumento con mejores propiedades psicométricas (Zaror et al., 2018b).
La región de la Araucanía se encuentra entre las regiones con más altos indicadores de pobreza y vulnerabilidad del país y junto con ello presenta también los peores índices de salud oral. Dado que lo recursos públicos son limitados, resulta de vital importancia conocer el impacto de las patologías orales en la CVRSO, con el fin de determinar prioridades según las patologías que más impactan en la calidad de vida de niños preescolares y que permitan implementar políticas públicas basadas en la perspectiva del paciente y sus familias. En respuesta a esto, el objetivo de este estudio fue determinar el impacto de las variables sociodemográficas y clínicas en la CVRSO en preescolares de la Comuna de Temuco, Chile.

\section{MATERIAL Y MÉTODO}

Se realizó un estudio de corte transversal entre octubre de 2017 y noviembre de 2018 en niños que asisten a jardines infantiles de INTEGRA en la ciudad de Temuco, Región de La Araucanía. El objetivo de la Fundación INTEGRA es ampliar la cobertura educativa y proporcionar alimentación a preescolares de bajo nivel socioeconómico. El proyecto fue evaluado y aprobado por el comité de ética científico de la Universidad de La Frontera, Temuco (Folio $N^{\circ} 020 \_17$ ) y este informe fue preparado de acuerdo con las recomendaciones de Strengthening the Reporting of Observational Studies in Epidemiology (STROBE) (von Elm et al., 2008).

Se incluyeron preescolares entre 3 a 5 años inscritos en los jardines de Fundación INTEGRA de la ciudad de Temuco, cuyos padres firmaron el consentimiento informado. Se excluyeron aquellos preescolares con enfermedades sistémicas, medicación crónica o en situación de discapacidad. El universo de la población fue 673 preescolares entre 36 hasta 71 meses de edad, de quince establecimientos educacionales de la Fundación INTEGRA en Temuco. Se invitaron a participar a todos los padres de los niños que cumplieran con los criterios de inclusión.

El examen clínico fue realizado por 4 investigadores previamente calibrados (correlación interobservador kappa $=0,83$ y correlación intraobservador kappa $=0,94$ ) en salas adaptadas para el estudio en dependencias de los establecimientos educacionales incluidos. Los exámenes se realizaron bajo luz LED portátil e inspección visual sistematizada de la cavidad oral, para lo cual se utilizó espejo dental y explorador para eliminar placa bacteriana. 
El diagnóstico de caries se realizó de acuerdo a los criterios propuestos por la OMS (World Health Organization, 2013). Para la variable defectos del desarrollo del esmalte (DDE) se consideró el índice de desarrollo de defectos del esmalte (DE) modificado de acuerdo con la encuesta de Salud Oral (World Health Organization). Los traumatismos dentoalveolares (TDA) fueron identificados mediante los criterios de la Asociación Internacional de Traumatología Dentaria (IADT) (Malmgren et al., 2012). La maloclusión fue evaluada clínicamente según la presencia o ausencia de al menos una de las siguientes anomalías: mordida abierta anterior, resalte mayor a $4 \mathrm{~mm}$ y mordida invertida o mordida cruzada. La higiene oral se determinó mediante un cuestionario dirigido a las familias, en el que se in indicó la cantidad de veces en que se realizaba la higiene oral por un adulto.Se definió como insuficiente cuando se realizaban menos de 2 cepillados diarios. La observación directa de placa visible en la superficie vestibular de los dientes primarios se registró como presencia o ausencia en la ficha clínica. La gingivitis se evaluó de acuerdo con los criterios propuestos por la OMS para evaluar estado periodontal (World Health Organization).

La calidad de vida relacionada a salud oral fue evaluada a través de la versión en español del cuestionario Early Childhood Oral Health Impact Scale (ECOHIS), validado en población chilena (Zaror et al., 2018a). Éste se compone de 13 ítems con dos secciones principales: la primera que mide el impacto en el niño y la segunda en la familia. La sección de impacto infantil, tiene 4 dominios: sintomático, funcional, psicológico y autoimagen e interacción social. La sección de impacto familiar presenta 2 dominios: angustia de los padres y función familiar. El cuestionario se califica utilizando una escala tipo Likert de cinco puntos, con respuestas que van desde "Nunca" a "Muy a menudo" (equivalente a puntuaciones de 0 a 4). Los puntajes de ECOHIS son calculados como una suma de cada una de las respuestas para las secciones del niño y familia por separado y también como un puntaje total. Por lo tanto, la puntuación total oscila entre 0 a 52 , donde las puntuaciones más altas indican un mayor impacto en la CVRSO.

Se solicitó a todos los padres que completaran un cuestionario estructurado al comienzo del estudio para recopilar información sobre los antecedentes sociodemográficos del niño y otros antecedentes relevantes de salud de los menores. El nivel socioeconómico se calculó mediante la cobertura de seguro de salud del preescolar, siguiendo los parámetros establecidos por el Fondo Nacional de Salud de Chile (FONASA), que clasifica a sus beneficiarios en diferentes categorías según sus ingresos. A: personas sin hogar, indigentes o extremadamente pobres; B: personas con un ingreso imponible mensual menor o igual a CLP 250,000 (USD \$ 370); C: personas con un ingreso imponible mensual mayor a CLP 250,000 (USD \$ 370) y menor o igual a CLP 365,000 (USD \$ 545) y D: personas con un ingreso imponible mensual superior a CLP 365,000 (USD \$ 545) (Superintendencia de Salud, 2016).

Posterior a la realización de los exámenes odontológicos, se entregó un informe a los padres o tutor principal de los niños con el estado de salud oral del menor y recomendaciones relativas al estado oral individual de cada uno. Todos los niños participantes recibieron un cepillo dental, una vez finalizado su examen dental.

Con respecto al análisis estadístico, se condujo un análisis descriptivo con la distribución de la ECOHIS, las características sociodemográfica y clínicas de la muestra.

Dada la distribución asimétrica de la puntuación de la ECOHIS, debido a una alta frecuencia de ceros, la comparación entre las variables clínicas y sociodemográficas con CVRSO fue realizada a través de las pruebas no paramétricas de Kruskal-Wallis para variables de independientes dicotómicas (oclusión, TDA, hipoplasia posterior, hipoplasia anterior, placa visible, gingivitis, aseo oral, lactancia materna actual, sexo apoderados, sexo de pacientes, previsión) o Mann Whitney para variables de independiente categóricas (caries, estado civil). Para la cuantificación de la variable caries, se categorizaron en el análisis 3 grupos principales: ceod 0; ceod entre 1 y menor o igual a 5 y ceod mayor o igual a 6 . En ambos casos se consideró un nivel de significación de 0,05.

Se llevo a cabo un análisis multivariado utilizando un modelo lineal generalizado. Este modelo permite suponer que la variable dependiente sigue distribuciones de probabilidad distintas de la distribución normal, como en este caso que sigue una distribución de Poisson. Se incluyeron en el modelo todas aquellas variables que presentaron un valor $p>0.2$ en el análisis bivariado. Finalmente se calcularon las razones de prevalencia (RP) con sus respectivos intervalos de confianza del $95 \%$. El análisis estadístico de los datos se llevó a cabo por medio del programa Stata 15 (Stata Corp, College Station, TX, USA). 


\section{RESULTADOS}

Se examinaron clínicamente un total de 300 preescolares, de los cuales 202 apoderados contestaron y entregaron la escala de impacto ECOHIS, lo que corresponde una tasa de respuesta de un $67.3 \%$. De los cuestionarios recibidos, todos siguieron las instrucciones para su llenado correcto y ninguno fue excluido del análisis de datos. El 91 $\%$ de los cuestionarios fue respondido por las madres o cuidadoras principales. Para los preescolares la edad promedio (desviación estándar, DE) fue 3,34 (DE 0,61) años y la edad promedio del cuidador principal fue 30,02 (DE 6,43) años. La Tabla I muestra la distribución de las variables socio-demográficas y clínicas. Destaca que el $30.2 \%$ de los niños evaluados presenta la enfermedad con distintos grados de severidad.

En la Tabla II se muestra la distribución de las medias de la ECOHIS. Las puntuaciones totales de ECOHIS variaron de 0 a 29 con una media de 2,60 (DE 4,64). Se muestra un impacto levemente mayor en el niño que en la familia (media 1,31 vs 1.29). La media más alta fue de 0.9 en el dominio de angustia parental y la más baja se obtuvo en el dominio autoimagen e interacción social con una media de 0,03 . Dentro de las preguntas de la ECOHIS, se observa que las preguntas relacionadas con el dolor $(9,91 \%)$, la dificultad para comer $(3,47 \%)$, la dificultad para pronunciar palabras $(3,48 \%)$, el ausentismo escolar $(3,48 \%)$ y problemas al dormir $(3,47 \%)$ fueron las reportadas con mayor frecuencia en la sección de impacto infantil. Para la sección de impacto familiar, las preguntas relacionadas a la molestia de algún miembro de la familia por problemas dentales de los hijos $(14,36 \%)$ y la culpa de algún miembro de la familia por problemas dentales de los hijos (12,88 \%) fueron las que se reportaron con mayor frecuencia.

La Tabla III, muestra las medias de las puntuaciones totales y por dominio de la ECOHIS según variables clínicas estudiadas. Para las variables oclusión, TDA y gingivitis, no se aprecia un impacto sobre la CVRSO en el valor total de la ECOHIS ni en sus do-
Tabla I. Características demográficas y clínicas de los niños participantes del estudio.

\begin{tabular}{|c|c|c|}
\hline Variables & $\mathbf{N}^{\circ}$ & $\%$ \\
\hline Sexo Pacientes & 202 & \\
\hline Hombres & 105 & 51,98 \\
\hline Mujeres & 97 & 48,02 \\
\hline \multicolumn{3}{|l|}{ Edad Pacientes } \\
\hline 3 años & 143 & 70,79 \\
\hline 4 años & 50 & 24,75 \\
\hline 5 años & 9 & 4,46 \\
\hline Sexo Padres & 202 & \\
\hline Ho mbres & 18 & 8,91 \\
\hline Mujeres & 184 & 91,09 \\
\hline \multicolumn{3}{|l|}{ Edad Cuidador Principal } \\
\hline Menor a 20 & 1 & 0,49 \\
\hline Entre 20 y 29 & 110 & 54,46 \\
\hline Entre 30 y 39 & 74 & 36,63 \\
\hline Mayor o igual a 40 & 17 & 8,42 \\
\hline \multicolumn{3}{|c|}{ Seguro de Salud / Nivel Socioeconómico } \\
\hline FONASA A & 94 & 46,53 \\
\hline FONASA B & 42 & 20,79 \\
\hline FONASA C & 30 & 14,85 \\
\hline FONASA D & 22 & 10,89 \\
\hline ISAPRE/Particular & 14 & 6,93 \\
\hline \multicolumn{3}{|l|}{ Estado Civil Padres } \\
\hline Soltera(o) & 139 & 68,81 \\
\hline Casada(o) & 58 & 28,71 \\
\hline Divorciada(o) & 4 & 1,98 \\
\hline Viuda(o) & 1 & 0,50 \\
\hline \multicolumn{3}{|l|}{ Caries } \\
\hline Ausente & 141 & 69,8 \\
\hline Presente & 61 & 30,2 \\
\hline \multicolumn{3}{|l|}{ ceod } \\
\hline ceod $=0$ & 141 & 69,80 \\
\hline ceod $>1<5$ & 48 & 23,76 \\
\hline ceod $>5$ & 13 & 6,44 \\
\hline \multicolumn{3}{|c|}{ Defectos del desarrollo del esmalte } \\
\hline Ausente & 172 & 85,15 \\
\hline Presente & 30 & 14,85 \\
\hline \multicolumn{3}{|l|}{ Hipoplasias Anteriores } \\
\hline Ausente & 182 & 90,1 \\
\hline Presente & 20 & 9,9 \\
\hline \multicolumn{3}{|l|}{ Hipoplasias Posteriores } \\
\hline Ausente & 176 & 87.13 \\
\hline Presente & 26 & 12,87 \\
\hline \multicolumn{3}{|l|}{ Placa Visible } \\
\hline Ausente & 85 & 42,08 \\
\hline Presente & 117 & 57,92 \\
\hline \multicolumn{3}{|l|}{ Gingivitis } \\
\hline Ausente & 101 & 50 \\
\hline Presente & 101 & 50 \\
\hline \multicolumn{3}{|l|}{ Maloclusión } \\
\hline Ausente & 134 & 66,34 \\
\hline Presente & 68 & 33.66 \\
\hline \multicolumn{3}{|c|}{ Traumatismo Dentoalveolar } \\
\hline Ausente & 176 & 86,63 \\
\hline Presente & 26 & 13,37 \\
\hline
\end{tabular}


Tabla II. Media, desviación estándar y rango observado para el total de la ECOHIS, secciones y dominios.

\begin{tabular}{lcc}
\hline ECOHIS Total, dominios e impacto & Media (DE) & Rango Observado \\
\hline Impacto Infantil & $1,31(2,65)$ & $0-17$ \\
Dominio Sintomático & $0,41(0,78)$ & $0-4$ \\
Dominio Funcional & $0,63(1,47)$ & $0-9$ \\
Dominio Psicológico & $0,24(0,73)$ & $0-6$ \\
Dominio Autoimagen e interacción social & $0,03(0,24)$ & $0-2$ \\
Impacto Familiar & $1,29(2,59)$ & $0-16$ \\
Dominio Angustia de los padres & $0,90(1,79)$ & $0-8$ \\
Dominio Funcionamiento Familiar & $0,39(1,15)$ & $0-8$ \\
ECOHIS Total & $2,60(4,64)$ & $0-29$ \\
\hline
\end{tabular}

$\mathrm{DE}=$ desviación estándar

Tabla III. Media y desviación estándar (DE) de los dominios de ECOHIS según variables clínicas.

\begin{tabular}{|c|c|c|c|c|c|c|c|c|c|}
\hline & $\begin{array}{c}\text { ECOHIS } \\
\text { (total) }\end{array}$ & $\begin{array}{l}\text { Dominio: } \\
\text { Sintomático }\end{array}$ & $\begin{array}{l}\text { Dominio: } \\
\text { Funcional }\end{array}$ & $\begin{array}{l}\text { Dominio: } \\
\text { Psicológico }\end{array}$ & $\begin{array}{l}\text { Dominio: } \\
\text { Social }\end{array}$ & $\begin{array}{l}\text { Dominio: } \\
\text { Angustia de } \\
\text { los padres }\end{array}$ & $\begin{array}{l}\text { Dominio: } \\
\text { Funcionamiento } \\
\text { Familiar }\end{array}$ & $\begin{array}{l}\text { Im pacto } \\
\text { Infantil }\end{array}$ & $\begin{array}{l}\text { Impacto } \\
\text { Familiar }\end{array}$ \\
\hline & Media (DE) & Media (DE) & Media (DE) & Media (DE) & Media (DE) & Media (DE) & Media (DE) & Media (DE) & Media (DE) \\
\hline \multicolumn{10}{|l|}{ Caries } \\
\hline ceod $=0$ & $1,73(2,78)$ & $0,32(0,67)$ & $0,41(0,97)$ & $0,16(0,47)$ & $0(0)$ & $0,53(1,25)$ & $0,30(0,96)$ & $0,89(1,64)$ & $0,84(1,93)$ \\
\hline ceod $>1<5$ & $2,63(3,98)$ & $0,38(0,61)$ & $0,72(1,38)$ & $0,13(0,44)$ & $0,08(0,40)$ & $1,06(1,83)$ & $0,25(0,67)$ & $1,31(2,42)$ & $1,31(2,16)$ \\
\hline ceod $>5$ & $11,92(10,27)$ & $1,46(1,51)$ & $2,69(3,57)$ & $1,54(1,90)$ & $0,15(0,55)$ & $4,23(2,95)$ & $1,84(2,67)$ & $5,86(6,24)$ & $6,08(4,87)$ \\
\hline Normal & $2,42(4,41)$ & $0,4(0,81)$ & $0,64(1,49)$ & $0,24(0,65)$ & $0,03(0,24)$ & $0,79(1,56)$ & $0,33(0,99)$ & $1,30(2,73)$ & $1,13(2,19)$ \\
\hline Alterado & $2,94(5,09)$ & $0,42(0,72)$ & $0,63(1,45)$ & $0,25(0,88)$ & $0,03(0,24)$ & $1,10(2,18)$ & $0,51(1,41)$ & $1,33(2,51)$ & $1,61(3,25)$ \\
\hline valor $p$ & 0,218 & 0,590 & 0,851 & 0,863 & 0,990 & 0,574 & 0,404 & 0,603 & 0,322 \\
\hline \multicolumn{10}{|l|}{ TDA } \\
\hline Ausente & $2,65(4,76)$ & $0,40(0,76)$ & $0,61(1,47)$ & $0,26(0,75)$ & $0,03(0,26)$ & $0,93(1,83)$ & $0,42(1,21)$ & $1,31(2,67$ & $1,35(2,69)$ \\
\hline Presente & $2,23(3,82)$ & $0,42(0,90)$ & $0,77(1,53)$ & $0,15(0,54)$ & $0,03(0,24)$ & $0,69(1,52)$ & $0,19(0,57)$ & $1,35(2,61)$ & $0,88(1,77)$ \\
\hline valor $p$ & 0,569 & 0,939 & 0,722 & 0,366 & 0,504 & 0,327 & 0,459 & 0,987 & 0,345 \\
\hline \multicolumn{10}{|c|}{ Hipoplasia Posterior } \\
\hline Presente & $4,46(6,69)$ & $0,42(1,03)$ & $0,65(1,41)$ & $0,5(1,30)$ & $0,08(0,39)$ & $1,88(2,63)$ & $0,92(1,96)$ & $1,65(3,24)$ & $2,81(4,28)$ \\
\hline valor $p$ & 0,150 & 0,449 & 0,635 & 0,326 & 0,288 & 0,016 & 0,082 & 0,998 & $0,017^{*}$ \\
\hline \multicolumn{10}{|c|}{ Hipoplasia Anterior } \\
\hline Ausente & $0,27(4,18)$ & $0,37(0,72)$ & $0,62(1,48)$ & $0,20(0,60)$ & $0,03(0,26)$ & $0,74(1,57)$ & $0,30(0,93)$ & $1,23(2,59)$ & $1,04(2,12)$ \\
\hline Presente & $5,55(7,17)$ & $0,7(1,17)$ & $0,7(1,42)$ & $0,65(1,42)$ & $0(0)$ & $2,3(2,85)$ & $1,2(2,21)$ & $2,05(3,17)$ & $2,5(4,74)$ \\
\hline valor $p$ & $\mathbf{0 , 0 1 3 ^ { * }}$ & 0,283 & 0,410 & $0,027^{*}$ & 0,564 & $0,002^{\star}$ & $0,0421^{*}$ & 0,107 & $0,006^{\star}$ \\
\hline \multicolumn{10}{|l|}{ Placa Visible } \\
\hline Ausente & $1,93(3,14)$ & $0,24(0,57)$ & $0,58(1,07)$ & $0,13(0,43)$ & $0,05(0,30)$ & $0,48(1,01)$ & $0,4(1,10)$ & $1,05(1,91)$ & $0,88(1,94)$ \\
\hline Presente & $4,09(5,44)$ & $0,49(0,90)$ & $0,68(1,71)$ & $0,32(0,88)$ & $0,02(0,18)$ & $1,20(2,14)$ & $0,38(1,18)$ & $1,50(3,08)$ & $1,58(2,95)$ \\
\hline $\begin{array}{l}\text { valor } p \\
\text { Gingivitis }\end{array}$ & 0,080 & 0,080 & 0,640 & 0,060 & 0,390 & $0,005^{*}$ & 0,930 & 0,230 & 0,058 \\
\hline Ausente & $2,23(2,80)$ & $0,36(0,72)$ & $0,52(1,15)$ & $0,17(0,49)$ & $0,04(0,28)$ & $0,70(1,43)$ & $0,44(1,17)$ & $1,09(2,13)$ & $1,14(2,38)$ \\
\hline Presente & $2,97(5,34)$ & $0,46(0,84)$ & $0,74(1,74)$ & $0,32(0,90)$ & $0,02(0,20)$ & $1,09(2,07)$ & $0,35(1,13)$ & $1,53(3,08)$ & $1,44(2,79)$ \\
\hline Valor $p$ & 0,464 & 0,418 & 0,971 & 0,370 & 0,562 & 0,339 & 0,746 & 0,463 & 0,240 \\
\hline
\end{tabular}

* Estadísticamente significativo en $\mathrm{p}<0.05 \mathrm{ceod}=$ Índice de dientes cariados, perdidos y obturados.

minios. En relación a caries dental, se observa que ésta presenta un impacto en todos los dominios de la escala $(p=<0,001)$, observándose puntajes más altos de la ECOHIS en pacientes que presentan mayor cantidad de lesiones de caries. Mientras mayor es la severidad de la caries, mayor es el impacto en la CVRSO. Para pacientes libres de caries en el valor total de ECOHIS la media observada fue 1,73 (DE 2,78) versus la media obtenida en pacientes con 6 lesiones de caries o más que fue de 11,92 (DE 10,27).

La Tabla IV, muestra la asociación entre variables clínicas y sociodemográficas ajustadas y no ajustadas. El análisis bivariado, muestra que las variables estado civil, índice ceod, placa visible e hipoplasias anteriores y posteriores se asociaron significativamente 
NÚÑEZ-CONTRERAS, J.; HOFER-DURÁN, P.; SINSAY-SCHMEISSER, J. \& ZAROR, C. Impacto de las condiciones sociodemográficas y orales en la calidad de vida relacionada a la salud oral en preescolares de Temuco, Chile. Int. J. Odontostomat., 15(2):503-512, 2021.

con la puntuación total de la ECOHIS para calidad de vida $(p<0,05)$.

En el análisis multivariado, los valores ajustados muestran que el estado civil viudo(a) $(P R=2,93 ; p=$
$<0,001)$ y un ceod $>$ a $5(P R=7,16 ; p=<0,001)$, muestran un impacto negativo sobre la calidad de vida relacionada a la salud oral, sin embargo, al calcular los valores ajustados, placa visible e hipoplasias anteriores y posteriores, no muestran impacto en la CVRSO.

Tabla IV. Asociación entre variables clínicas y sociodemográficas ajustadas y no ajustadas.

\begin{tabular}{|c|c|c|c|c|c|c|}
\hline \multirow{2}{*}{$\overline{\text { Variable }}$} & \multicolumn{3}{|c|}{ Valores no ajus tados } & \multicolumn{3}{|c|}{ Valores ajustados* } \\
\hline & $\mathrm{PR}$ & IC $95 \%$ & valor $p$ & $\mathrm{PR}$ & IC $95 \%$ & valor $p$ \\
\hline \multicolumn{7}{|l|}{ Edad pacientes } \\
\hline Años & 1,28 & $0,92-1,80$ & 0,143 & 1,17 & $0,84-1,62$ & 0,351 \\
\hline \multicolumn{7}{|l|}{ Edad apoderados } \\
\hline Años & 1,00 & $0,96-1,05$ & 0,906 & & & \\
\hline \multicolumn{7}{|l|}{ Sexo pacientes } \\
\hline Hombre & 1 & & & 1 & & \\
\hline Mujeres & 0,69 & $0,43-1,11$ & 0,126 & 0,72 & $0,48-1,08$ & 0,114 \\
\hline \multicolumn{7}{|l|}{ Sexo apoderados } \\
\hline Hombres & 1 & & & & & \\
\hline Mujeres & 0,97 & $0,51-1,87$ & 0,933 & & & \\
\hline \multicolumn{7}{|l|}{ Previsión } \\
\hline Fonasa A & 1 & & & & & \\
\hline Fonasa B-C-D e Isapre & 0,64 & $0,40-1,04$ & 0,077 & 0,72 & $0,47-1,10$ & 0,127 \\
\hline \multicolumn{7}{|l|}{ Estado civil apoderados } \\
\hline Soltero & 1 & & & 1 & & \\
\hline Casado & 0,79 & $0,48-1,32$ & 0,379 & 1,19 & $0,70-1,2$ & 0,519 \\
\hline Separado & 0,63 & $0,22-1,79$ & 0,388 & 1,29 & $0,46-3,65$ & 0,632 \\
\hline Viudo(a) & 1,44 & $1,06-1,95$ & $0,019^{*}$ & 2,93 & $1,88-4,58$ & $<0,001^{*}$ \\
\hline \multicolumn{7}{|l|}{ Índice ceod } \\
\hline ceod $=0$ & 1 & & & 1 & & \\
\hline ceod $>1<5$ & 1,51 & $0,92-2,50$ & 0,103 & 1,38 & $0,82-2,34$ & 0,229 \\
\hline ceod $\geq 5$ & 6,89 & $4,08-11,62$ & $<0,001^{*}$ & 7,16 & $4,18-12,25$ & $<0,001^{*}$ \\
\hline \multicolumn{7}{|l|}{ Oclusión } \\
\hline Oclusión Normal & 1 & & & & & \\
\hline Oclusión Alterada & 1,21 & $0,72-1,79$ & 0,467 & & & \\
\hline \multicolumn{7}{|l|}{ TDA } \\
\hline Ausencia de TDA & 1 & & & & & \\
\hline Presencia de TDA & 0,84 & $0,42-1,69$ & 0,627 & & & \\
\hline \multicolumn{7}{|l|}{ Placa Visible } \\
\hline Ausencia de Placa Visible & 1 & & & 1 & & \\
\hline Presencia de Placa Visible & 1,60 & $1,00-2,56$ & $0,050^{*}$ & 0,96 & $0,60-1,54$ & 0,866 \\
\hline \multicolumn{7}{|l|}{ Gingivitis } \\
\hline Ausencia de gingivitis & 1 & & & & & \\
\hline Presencia de Gingivitis & 1,33 & $0,82-2,16$ & 0,243 & & & \\
\hline \multicolumn{7}{|l|}{ Hipoplasia Anterior } \\
\hline Ausencia de Hipoplasia Anterior & 1 & & & 1 & & \\
\hline Presencia de Hipoplasia Anterior & 2,44 & $1,32-4,51$ & $0,004^{*}$ & 1,73 & $0,74-4,04$ & 0,204 \\
\hline \multicolumn{7}{|l|}{ Hipoplasia Posterior } \\
\hline Ausencia de Hipoplasia Posterior & 1 & & & 1 & & \\
\hline Presencia de Hipoplas ia Posterior & 1,91 & $1,02-3,59$ & $0,041^{*}$ & 1,22 & $0,56-2,70$ & 0,615 \\
\hline \multicolumn{7}{|l|}{ Aseo bucal } \\
\hline Aseo bucal adecuado & 1 & & & 1 & & \\
\hline Aseo bucal deficiente & 2,17 & $0,67-7,06$ & 0,196 & 0,61 & $0,30-1,25$ & 0,178 \\
\hline \multicolumn{7}{|l|}{ Lactancia Materna Actual } \\
\hline Ausencia de lactancia materna & 1 & & & & & \\
\hline Presencia de lactancia materna & 1,1 & $0,44-2,73$ & 0,842 & & & \\
\hline
\end{tabular}




\section{DISCUSIÓN}

Este estudio evaluó el impacto de diferentes variables sociodemográficas y clínicas en la CVRSO en una población de preescolares de la ciudad de Temuco, Chile, pudiendo evidenciarse que la caries dental y el vivir con un cuidador viudo, se asociaron directamente a una disminución en la calidad de vida de los pacientes, no así las maloclusiones, traumatismos dentoalveolares, hipoplasias posteriores y anteriores del esmalte, placa visible y gingivitis.

Dentro de las variables sociodemográficas, nuestro estudio no encontró asociación entre el estatus socioeconómico y la disminución en la CVRSO, resultados que concuerdan con lo ya publicado por otros autores (Goettems et al., 2011; Kramer et al., 2013; Abanto et al., 2015), sin embargo, existen quienes proponen que la disminución de la CVRSO estaría asociada a desventajas socioeconómicas, al influir en la capacidad de respuesta de las familias a los problemas de salud oral. (Abanto et al., 2010; Chaffee et al.). Para este estudio evaluamos nivel socioeconómico calculado mediante la cobertura de seguro de salud chileno, sin embargo, esto pudiese ser confundente, ya que solo refleja el ingreso del grupo familiar completo y no el ingreso per cápita de cada familia, lo que podría hablar de las diferencias entre nuestros resultados y lo publicado en la literatura (Chaffee et al.; Ramos-Jorge et al., 2015).

Respecto del estado civil del cuidador, encontramos una disminución de la calidad de vida cuando el estado civil del cuidador principal es viudo(a), sin embargo los estados civiles solteros, casados o separados no se vieron asociados a una disminución en la CVRSO de los menores. La literatura hasta el momento solo ha evaluado estos últimos 3 estados civiles, no encontrando asociaciones (Abanto et al., 2010; MartinsJúnior et al., 2013). Esto fue confirmado posteriormente por Kumar et al. (2014) en su revisión sistemática sobre influencia de factores sociodemográficos en la CVRSO. Sin embargo, tampoco evalúa como variable a aquellos cuidadores viudos, lo que podría explicarse debido a la baja frecuencia de madres o padres viudas(os) de niños en edad preescolar. Este hallazgo deber ser considerado con cautela y debe ser estudiado mediante métodos apropiados en futuras investigaciones.

En nuestro estudio, los niños cuyo ceod fue mayor a 5, tuvieron 7,16 veces mas probabilidades de ver afectada su calidad de vida, en comparación con los niños libres de caries, siendo esto consistente con la literatura (Gomes et al., 2014; Abanto et al., 2015, 2016, 2018; Kramer et al.; Ramos-Jorge et al., 2014; CorrêaFaria et al., 2016; Fernandes et al., 2017; Ramos-Jorge et al., 2015; Pesaressi et al., 2020). La caries dental es la enfermedad crónica mas común en niños y aquellos que la experimentan de forma mas severa, muchas veces presentan dolor, u otras alteraciones de la función, explicando así la percepción negativa que pudiesen tener los padres o los mismos niños (Scarpelli et al., 2013; Guedes el al., 2016). De igual manera, la caries dental ha demostrado tener un impacto negativo en la apariencia estética, principalmente relacionada a las interacciones psicosociales de los menores (Abanto et al., 2010; Ramos-Jorge et al., 2014). Otro efecto de la enfermedad que puede ser considerado es que debido a las experiencias asociadas a dolor en los niños se generan situaciones complejas en el entorno familiar, como tener que pedir permiso en el trabajo para cuidar de la salud oral de sus hijos y muchas veces incurrir en gastos adicionales para atenciones dentales (Pahel el al., 2007; Guedes et al.). En algunos artículos también se menciona la culpabilidad que sienten los padres, por la presencia de enfermedades orales, siendo el dolor uno de los factores que produce este sentimiento en ellos (Abanto et al., 2010; Gomes et al.; Guedes et al.), junto con el miedo de ser juzgados por los problemas de salud oral de sus hijos (Carvalho et al., 2012). Este fenómeno se podría deber a que las causas de la caries dental o de otras enfermedades orales se encuentran altamente difundidas actualmente, por tanto, si los padres conocen como prevenir las enfermedades orales es razonable pensar, que la falta en la ejecución de medidas preventivas puede llevar a estos sentimientos de culpa

Nuestro estudio no encontró una asociación entre la presencia de maloclusiones y la disminución de la calidad de vida, lo que concuerda con lo expresado en la literatura (Abanto et al., 2010; Carvalho et al., 2013; Corrêa-Faria et al.; Gomes et al.; Kragt et al., 2016; Scarpelli et al.). Esto se puede explicar debido a la sicología del desarrollo en los niños, donde a partir de los 6 años recién comienza el desarrollo del pensamiento abstracto y el concepto de la imagen propia, lo que los lleva a compararse a mayor edad con otros niños tanto físicamente, como en otras características de su personalidad (Abanto et al., 2010; Cameron \& Widmer, 2013). Es por este motivo, que los investigadores han encontrado que en niños desde los 8 años la CVRSO se puede ver disminuida por las maloclusiones, viéndose el mayor impacto entre los 11 y los 14 años (Kragt et al.). Otros estudios relacionan la percepción de los padres de los dientes prima- 
NÚÑEZ-CONTRERAS, J.; HOFER-DURÁN, P.; SINSAY-SCHMEISSER, J. \& ZAROR, C. Impacto de las condiciones sociodemográficas y orales en la calidad de vida relacionada a la salud oral en preescolares de Temuco, Chile. Int. J. Odontostomat., 15(2):503-512, 2021.

rios como algo transitorio, por ende no le asignarían tanta importancia (Carvalho et al., 2013).

No se encontraron asociación entre TDA y la disminución de CVRSO, lo que se condice con previos artículos(Abanto et al., 2010, 2014; Fernandes et al.; Scarpelli et al.; Vieira-Andrade et al., 2015), pudiéndose atribuir al tipo de trauma que se encuentra con mas frecuencia, que son las fracturas de esmalte y en muchas ocasiones los padres no buscan tratamiento frente a ellas, o no son capaces de detectar traumas de este tipo (Ramos-Jorge et al., 2013; Scarpelli et al.; Lopez et al., 2019). En este aspecto la literatura no es unánime. Borges et al. (2017) en su revisión sistemática y meta-análisis concluye que los TDA impactan la calidad de vida de los preescolares, pero no en los padres. Por otro lado, debe tenerse en consideración la severidad del traumatismo dentoalveolar. Evidencia muestra que los TDA severos se asocian a un mayor impacto en la CVRSO (Borges et al.; Zaror et al., 2018a), ya que muchos casos complejos de TDA requieren de atenciones complejas, largas y los TDA pueden afectar la función y generar dolor en los menores (Aldrigui et al., 2011; Gomes et al.; Abanto et al., 2015; Firmino et al., 2016; Kramer et al.). De igual manera, la literatura propone cautela respecto al uso de la ECOHIS para la evaluación de las maloclusiones y TDA. Si bien, esta ha sido validada para evaluar el impacto de problemas de salud oral en general (Zaror et al., 2018b) las preguntas del cuestionario fueron creadas para evaluar la caries dental, más que otros problemas de salud oral (Abanto et al., 2010; Carvalho et al., 2013).

Los DDE tampoco mostraron tener un impacto negativo en la CVRSO. Estudios señalan que podría deberse a la falta de conocimiento respecto de los DDE por parte de los padres, por ende, como la ECOHIS es respondida por los padres o cuidadores y se basa en su percepción de la salud oral de los niños, dan mas atención a otro tipo de problemas que impliquen impotencia funcional, dolor o cambios en las interacciones sociales (Corrêa-Faria et al.). A nuestro conocimiento hay muy pocos estudios donde se evalúe la asociación de defectos del desarrollo del esmalte en población preescolar (Babu et al., 2017; Corrêa-Faria et al.; Scarpelli et al.; Andrade et al., 2019) siendo las principales dificultades para realizar comparaciones entre estudios las diferencias metodológicas relacionadas a los instrumentos para medir CVRSO, los dientes evaluados y el tipo de análisis estadísticos utilizados, por lo que los resultados aún son dispares. La magnitud de la alteración pudiese ser un factor decisivo en los efectos sobre la CVRSO, debido a la sensibilidad dental o el riesgo de caries aumen- tado que puede producir, así como también, posibles cambios en la coloración del diente y alteraciones de la morfología, pudiendo generar vergüenza y ansiedad en las interacciones sociales de los menores (Salanitri \& Seow, 2013; Vargas-Ferreira et al., 2015; Andrade et al.), por ende es un factor relevante a profundizar en próximas investigaciones.

Para las variables placa visible y gingivitis nuestro estudio no muestra un impacto negativo sobre la CVRSO. A nuestro conocimiento no existen estudios donde se evalúen estos parámetros en preescolares, sin embargo podemos pensar que si bien, la gingivitis es la enfermedad del periodonto mas común en niños y adolescentes, no genera dolor, lo que puede influir en que los padres no lo perciban como un problema. Además pudiese relacionarse a la percepción de los adultos de las enfermedades periodontales, ya que se ha evidenciado en estudios previos que los pacientes que padecen enfermedades del periodonto, no logran identificar sus síntomas y no comprenden el impacto de la enfermedad (Tascón et al., 2006).

La principal limitación del estudio es la homogeneidad de la muestra en relación al contexto social (nivel socioeconómico bajo/medio bajo), por lo que no se pueden generalizar los resultados a otros estratos socioeconómicos. Es importante considerar que el diagnóstico fue basado por los criterios establecidos por la Organización Mundial de la Salud, la cual no incluyó lesiones no cavitadas, lo que puede llevar a un subdiagnóstico de la enfermedad. Guedes et al. evaluaron este parámetro, concluyendo que efectivamente las lesiones no cavitadas no presentaban un impacto negativo en la calidad de vida de los menores, pudiendo estar relacionado a que este tipo de lesiones usualmente no presentan dolor o compromiso estético, por lo que muchas veces los padres o los niños no reporten afectación para dicho parámetro.

Los resultados del presente estudio muestran que vivir con cuidadores viudos y la severidad de la caries dental tiene un impacto negativo sobre la CVRSO de los preescolares y sus familias, no encontrándose asociación con otras variables sociodemográficas o clínicas. Estos hallazgos deben ser corroborados con una muestra más heterogénea a través de un estudio de cohorte que incluya otras variables confusoras, como nivel educacional de los padres, ingreso familiar o vulnerabilidad de los menores, así como también, a través de métodos cualitativos que permitan mejorar el entendimiento de como diferentes factores impactan la CVRSO en preescolares. 
NÚÑEZ-CONTRERAS, J.; HOFER-DURÁN, P.; SINSAYSCHMEISSER, J. \& ZAROR, C. Impact of Sociodemographic and Oral Conditions on Oral Health Related Quality of Life in Preschool Children From Temuco, Chile. Int. J. Odontostomat., 15(2):503-512, 2021.

ABSTRACT: Oral conditions are highly prevalent and affect the oral health related quality of life (OHRQoL) of preschool children. The aim of this study was to determine the impact of sociodemographic and oral conditions on OHRQoL in preschool children from Temuco, Chile. A cross-sectional study was carried out in preschool children between 3 and 5 years of age who attend INTEGRA kindergartens in Temuco, Chile. The prevalence of caries, malocclusions, traumatic dental injuries, anterior and posterior hypoplasia, presence of plaque, and gingivitis was measured by four previously calibrated examiners. The OHRQoL was measured through the Spanish version of the Early Childhood Oral Health Impact Scale (ECOHIS). Parents also completed a questionnaire on the child's sociodemographic background. The bivariate association between clinical and sociodemographic variables with OHRQoL was carried out through non-parametric tests (Kruskal-Wallis or Mann Whitney). Subsequently, a multivariate analysis was performed using a general linear model to associate the different factors with OHRQoL. Two hundred and two preschoolers were included ( 105 boys and 97 girls) with a mean age of 3.3 (SD 0.6). The mean score and standard deviation (SD) of the total ECOHIS were 2.60 and 4.64 respectively. The multivariate analysis showed that preschool children who have widowed parents or caregivers $(P R=2.93$; $p=<0.001$ ) and a dmft index > to 5 (PR = 7.16; $p=<0.001$ ), had a negative impact on OHRQoL. No association was found with other sociodemographic or clinical variables. The presence of a dmft > 5 and living with widowed caregiver had a negative impact on the OHRQoL of preschool children and their parents.

preschool.

KEY WORDS: Oral health; quality of life, child,

\section{REFERENCIAS BIBLIOGRÁFICAS}

Abanto, J.; Carvalho, T. S.; Mendes, F. M.; Wanderley, M. T.; Raggio, D. P. \& Bönecker, M. Impact of oral diseases and disorders on oral health-related quality of life of preschool children. Community Dent. Oral Epidemiol., 39(2):105-14. 2010.

Abanto, J.; Paiva, S. M.; Sheiham, A.; Tsakos, G.; Mendes, F. M.; Cordeschi, T.; Vidigal, E. A. \& Bönecker, M. Changes in preschool children's OHRQoL after treatment of dental caries:responsiveness of the B-ECOHIS. Int. J. Paediatr. Dent., 26(4):259-65. 2016.

Abanto, J.; Panico, C.; Bönecker, M. \& Frazão, P. Impact of demographic and clinical variables on the oral health-related quality of life among five-year-old children: a population-based study using self-reports. Int. J. Paediatr. Dent., 28(1):43-51. 2018.

Abanto, J.; Tello, G.; Bonini, G. C.; Oliveira, L. B.; Murakami, C. \& Bönecker, M. Impact of traumatic dental injuries and malocclusions on quality of life of preschool children: a population-based study. Int. J. Paediatr. Dent., 25(1):18-28, 2015.

Abanto, J.; Tsakos, G.; Paiva, S. M.; Carvalho, T. S.; Raggio, D. P. \& Bönecker, M. Impact of dental caries and trauma on quality of life among 5-to 6-year-old children: perceptions of parents and children. Community Dent. Oral Epidemiol., 42(5):385-94. 2014.
Aldrigui, J. M.; Abanto, J.; Carvalho, T. S.; Mendes, F. M.; Wanderley, M. T.; Bönecker, M. \& Raggio, D. P. Impact of traumatic dental injuries and malocclusions on quality of life of young children. Health Qual. Life Outcomes., 9(1):78, 2011.

Andrade, N. S.; Dos Santos, I. T.; Lima, L.; Lima, C.; Moura, L.; Barros, S.; Moura. M. S. \& Lima, M. Impact of Developmental enamel defects on quality of life in 5-year-old children. Int. J. Paediatr. Dent., 29(5):557-65, 2019.

Babu, N. V.; Moureen, A. \& Bhanushali, P. V. Comparative Evaluation of Impact of Dental Caries, Malocclusion and Developmental Defects on Preschooler's Quality of Life. Int. J. Sci. Study, 4(11):1215, 2017.

Barbosa, T. \& Gavião, M. Oral health-related quality of life in children: Part II. Effects of clinical oral health status. A systematic review. Int. J. Dent. Hyg., 6(2):100-7, 2008.

Borges, T. S.; Vargas-Ferreira, F.; Kramer, P. F. \& Feldens, C. A. Impact of traumatic dental injuries on oral health-related quality of life of preschool children: A systematic review and metaanalysis. PLoS One, 12(2):1-13, 2017.

Cameron, A. C. \& Widmer, R. P. Handbook of Pediatric Dentistry. EBook. 4a ed. Elsevier Health Sciences, 2013.

Carvalho, A. C.; Paiva, S. M.; Viegas, C. M.; Scarpelli, A. C.; Ferreira F. M. \& Pordeus, I. A. Impact of malocclusion on oral health-related quality of life among Brazilian preschool children: a populationbased study. Braz. Dent. J., 24(6):655-61, 2013.

Carvalho, T. S.; Abanto, J.; Mendes, F. M.; Raggio, D. P. \& Bönecker M. Association between parental guilt and oral health problems in preschool children. Braz. Oral Res., 26(6):557-63, 2012.

Chaffee, B. W.; Rodrigues, P. H.; Kramer, P. F.; Vítolo, M. R. \& Feldens C. A. Oral health-related quality-of-life scores differ by socioeconomic status and caries experience. Community Dent. Oral Epidemiol., 45(3):216-24, 2017.

Corrêa-Faria, P.; Paixão-Gonçalves, S.; Paiva, S. M.; Martins-Júnior P. A.; Vieira-Andrade, R. G.; Marques, L. S. \& Ramos-Jorge, M. L. Dental caries, but not malocclusion or developmental defects, negatively impacts preschoolers' quality of life. Int. J. Paediatr. Dent., 26(3):211-9, 2016

Dimberg, L.; Arnrup, K. \& Bondemark, L. The impact of malocclusion on the quality of life among children and adolescents:A systematic review of quantitative studies. Eur. J. Orthod., 37(3):238-47, 2015.

Espinoza-Espinoza, G.; Muñoz-Millán, P.; Vergara-González, C.; Atala-Acevedo, C. \& Zaror, C. Prevalence of early childhood caries in non-fluoridated rural areas of Chile. J. Oral Res., 5(8):307-13, 2016.

Fernandes, I. B.; Pereira, T. S.; Souza, D. S.; Ramos-Jorge, J.; Marques, L. S. \& Ramos-Jorge, M. L. Severity of Dental Caries and Quality of Life for Toddlers and Their Families. Pediatr. Dent., 39(2):118-23, 2017.

Firmino, R. T.; Gomes, M. C.; Clementino, M. A.; Martins, C. C.; Paiva, S. M. \& Granville-Garcia, A. F. Impact of oral health problems on the quality of life of preschool children: a case-control study. Int. J. Paediatr. Dent., 26(4):242-9, 2016.

Goettems, M. L.; Ardenghi, T. M.; Romano, A. R.; Demarco, F. F. \& Torriani, D. D. Influence of maternal dental anxiety on oral healthrelated quality of life of preschool children. Qual. Life Res., 20(6):951-9, 2011

Gomes, M. C.; Pinto-Sarmento, T. C. A; Costa, E. M. M. d. B.; Martins C. C.; Granville-Garcia, A. F. \& Paiva, S. M. Impact of oral health conditions on the quality of life of preschool children and their families: a cross-sectional study. Health Qual. Life Outcomes, 12(1):55. 2014.

Guedes, R. S.; Ardenghi, T. M.; Piovesan, C.; Emmanuelli, B. \& Mendes, F. M. Influence of initial caries lesions on quality of life in preschool children: a 2-year cohort study. Community Dent. Oral Epidemiol., 44(3):292-300, 2016.

Jans-Muñoz, A.; Díaz-Meléndez, J.; Vergara-González, C. \& ZarorSánchez, C. Frecuencia y severidad de la hipomineralización molar incisal en pacientes atendidos en las clínicas odontológicas de la Universidad de La Frontera, Chile. Int. J. Odontostomat., 5(2):13340, 2011. 
Kassebaum, N. J.; Smith, A. G. C.; Bernabé, E.; Fleming, T. D.; Reynolds, A. E.; Vos, T.; Murray, C. J. L.; Marcenes, W. \& GBD 2015 Oral Health Collaborators. Global, regional, and national prevalence, incidence, and disability-adjusted life years for oral conditions for 195 countries, 1990-2015: a systematic analysis for the global burden of diseases, injuries, and risk factors. J. Dent. Res., 96(4):380-7, 2017.

Kragt, L.; Dhamo, B.; Wolvius, E. B. \& Ongkosuwito, E. M. The impact of malocclusions on oral health-related quality of life in children-a systematic review and meta-analysis. Clin. Oral Investig., 20(8):1881-94, 2016.

Kramer, P. F.; Feldens, C. A.; Helena Ferreira, S.; Bervian, J.; Rodrigues, P. H. \& Peres, M. A. Exploring the impact of oral diseases and disorders on quality of life of preschool children. Community Dent. Oral Epidemiol., 41(4):327-35, 2013.

Kumar, S.; Kroon, J. \& Lalloo, R. A systematic review of the impact of parental socio-economic status and home environment characteristics on children's oral health related quality of life. Health Qual. Life Outcomes, 12(1):41, 2014.

Lopez, D.; Waidyatillake, N.; Zaror, C. \& Mariño, R. Impact of uncomplicated traumatic dental injuries on the quality of life of children and adolescents:a systematic review and meta-analysis. BMC Oral Health, 19(1):224, 2019.

Malmgren, B.; Andreasen, J. O.; Flores, M. T.; Robertson, A.; DiAngelis, A. J.; Andersson, L.; Cavalleri, G.; Cohenca, N.; Day, P.; Hicks, M. L.; et al. International Association of Dental Traumatology guidelines for the management of traumatic dental injuries: 3 . Injuries in the primary dentition. Dent. Traumatol., 28(3):174-182, 2012.

Martins-Júnior, P. A.; Vieira-Andrade, R. G.; Corrêa-Faria, P.; OliveiraFerreira, F.; Marques, L. S. \& Ramos-Jorge, M. L. Impact of early childhood caries on the oral health-related quality of life of preschool children and their parents. Caries Res., 47(3):211-8, 2013.

Ministerio de Salud. Plan Nacional de Salud Bucal 2018-2030. Santiago de Chile, Ministerio de Salud, Gobierno de Chile, 2017.

Nora, A. D.; da Silva Rodrigues, C.; de Oliveira Rocha, R.; Soares, F. Z. M.; Minatel Braga, M. \& Lenzi, T. L. Is caries associated with negative impact on oral health-related quality of life of pre-school children? A systematic review and meta-analysis. Pediatr. Dent., 40(7):403-11, 2018.

Pahel, B. T.; Rozier, R. G. \& Slade, G. D. Parental perceptions of children's oral health:The Early Childhood Oral Health Impact Scale (ECOHIS). Health Qual. Life Outcomes, 5(1):6, 2007.

Pesaressi, E.; Villena, R. S. \& Frencken, J. E. Dental caries and oral health related quality of life of 3-year-olds living in Lima, Peru. Int. J. Paediatr. Dent., 30(1):57-65, 2020.

Petti, S.; Andreasen, J. O.; Glendor, U. \& Andersson, L. The fifth most prevalent disease is being neglected by public health organisations. Lancet Glob. Health, 6(10):e1070-1, 2018a.

Petti, S.; Glendor, U. \& Andersson, L. World traumatic dental injury prevalence and incidence, a meta-analysis - One billion living people have had traumatic dental injuries. Dent. Traumatol., 34(2):71-86, 2018b.

Ramos-Jorge, J.; Alencar, B. M.; Pordeus, I. A.; Soares, M. E. C.; Marques, L. S.; Ramos-Jorge, M. L. \& Paiva, S. M. Impact of dental caries on quality of life among preschool children: emphasis on the type of tooth and stages of progression. Eur. J. Oral Sci., 123(2):8895, 2015.

Ramos-Jorge, J.; Pordeus, I. A.; Ramos-Jorge, M. L.; Marques, L. S. \& Paiva, S. M. Impact of untreated dental caries on quality of life of preschool children: different stages and activity. Community Dent. Oral Epidemiol., 42(4):311-22, 2014.

Ramos-Jorge, M. L.; Ramos-Jorge, J.; Mota-Veloso, I.; Oliva, K. J.; Zarzar, P. M. \& Marques, L. S. Parents' recognition of dental trauma in their children. Dent. Traumatol., 29(4):266-71, 2013.

Salanitri, S. \& Seow, W. K. Developmental enamel defects in the primary dentition: aetiology and clinical management. Aust. Dent. J., 58(2):133-40, 2013.

Scarpelli, A. C.; Paiva, S. M.; Viegas, C. M.; Carvalho, A. C.; Ferreira, F. M. \& Pordeus, I. A. Oral health-related quality of life among
Brazilian preschool children. Community Dent. Oral Epidemiol., 41(4):336-44, 2013.

Sischo, L. \& Broder, H.L. Oral health-related quality of life: what, why, how, and future implications. J. Dent. Res., 90(11):1264-70, 2011.

Superintendencia de Salud. ¿Cómo se clasifican los beneficiarios de FONASA según su tramo?. Santiago de Chile, Superintendencia de Salud, Ministerio de Salud, Gobierno de Chile, 2016. Disponible en: http://www.supersalud.gob.cl/consultas/667/w3-article6304.html

Tascón, J. E.; Londoño, D.; Jaramillo, C.; Burbano, P.; Mesa, M. \& Hernández, T. Creencias, prácticas y necesidad de tratamiento periodontal en una población adulta en Cali, 2003. Colomb. Med., 37(3):196-202, 2006.

Vargas-Ferreira, F.; Salas, M. M. S.; Nascimento, G. G.; Tarquinio, S. B. C.; Faggion, C. M.; Peres, M. A.; Thomson, W. M. \& Demarco, F. F. Association between developmental defects of enamel and dental caries:A systematic review and meta-analysis. J. Dent., 43(6):619-28, 2015

Vieira-Andrade, R. G.; Siqueira, M. B. L.; Gomes, G. B.; D’Avila, S.; Pordeus, I. A.; Paiva, S. M. \& Granville-Garcia, A. F. Impact of traumatic dental injury on the quality of life of young children: a case-control study. Int. Dent. J. 65(5):261-8, 2015.

von Elm, E.; Altman, D. G.; Egger, M.; Pocock, S. J.; Gøtzsche, P. C.; Vandenbroucke, J. P. \& Initiative, S. The Strengthening the Reporting of Observational Studies in Epidemiology (STROBE) statement: guidelines for reporting observational studies. Ann. Intern. Med., 147(8):573-7, 2008.

WHOQOL Group. The World Health Organization quality of life assessment (WHOQOL): position paper from the World Health Organization. Soc. Sci. Med., 41(10):1403-9, 1995.

World Health Organization. Oral Health Surveys; Basic Methods. Ginebra, World Health Organization, 2013.

Zaror, C.; Atala-Acevedo, C.; Espinoza-Espinoza, G.; Muñoz-Millán, P.; Muñoz, S.; Martínez-Zapata, M. J. \& Ferrer, M. Cross-cultural adaptation and psychometric evaluation of the early childhood oral health impact scale (ECOHIS) in chilean population. Health Qual. Life Outcomes, 16(1):232, 2018a.

Zaror, C.; Martínez-Zapata, M. J.; Abarca, J.; Díaz, J.; Pardo, Y. \& Ferrer, M. Impact of traumatic dental injuries on quality of life in preschoolers and schoolchildren: A systematic review and meta-analysis. Community Dent. Oral Epidemiol., 46(1):88-101, 2017.

Zaror, C.; Pardo, Y.; Espinoza-Espinoza, G.; Pont, A.; Muñoz-Millan, P.; Martínez-Zapata, M. J.; Vilagut, G.; Forero, C. G.; Olatz, G.; Alonso, J.; et al. Assessing oral health-related quality of life in children and adolescents:a systematic review and standardized comparison of available instruments. Clin. Oral Investig., 23(1):65$79,2018 b$.

Dirección para correspondencia

A/Prof. Carlos Zaror

Manuel Montt \#112

Facultad de Odontología

Universidad de La Frontera

Temuco - CHILE

E-mail: carlos.zaror@ufrontera.cl

Javiera Núñez-Contreras

Manuel Montt \#112

Facultad de Odontología

Universidad de La Frontera

Temuco - CHILE

E-mail: javiera.nunez@ufrontera.cl 